\title{
EDITORIALS
}

\section{Looking for Quality under the Lamppost}

\author{
Laurence F. McMahon, Jr., MD, MPH ${ }^{1,2,4}$ and Michele Heisler, MD, MA ${ }^{7,3}$ \\ 'Division of General Medicine, School of Medicine, University of Michigan, Ann Arbor, MI, USA; ${ }^{2}$ Department of Health Management and \\ Policy, School of Public Health, University of Michigan, Ann Arbor, MI, USA; ${ }^{3}$ Department of Health Behavior and Health Education, School of \\ Public Health, University of Michigan, Veterans Health System, Ann Arbor, MI, USA; ${ }^{4}$ Ann Arbor, MI, USA.
}

J Gen Intern Med 23(11):1923-5

DOI: $10.1007 / \mathrm{s} 11606-008-0809-4$

(c) Society of General Internal Medicine 2008

$\mathrm{C}$ oncepts such as clinical complexity, multimorbidity, severity of illness, and disease burden seek to characterize the subset of patients whose severity and/or number of illnesses disproportionately affect their lives. We see many such patients in our clinics and in our hospitals. As the numbers of these patients grow, with escalating health care costs and utilization, it is increasingly urgent to answer the following questions: Are these clinically complex patients receiving quality care? Are they satisfied with their experience with the health care system? How can we best measure these important domains and ensure that quality measures adequately are directing providers and health systems to prioritize attention and resources to the most important areas for these patients? These issues, while important for all patients, are especially critical for clinically complex patients.

The paper by Werner and Chang both adds to our understanding of these issues as well as highlights some of the technical problems in attempting to answer questions of quality of care and patient satisfaction ${ }^{1}$. Werner and Chang build on recent work on the impact of performance measurement on clinically complex patients in two important ways. Several recent papers have documented that clinically complex patients are more likely than less complex patients to receive recommended care $^{2,3}$, but in prior research frequency of medical visits was either not assessed or, in at least one study $^{3}$, did not affect the independent relationship between multimorbidity and quality of care. In contrast, Werner and Change found that higher quality of care was mediated by increased frequency of medical visits. In multivariate analyses controlling for visit frequency, higher clinical complexity was associated with lower odds of receiving recommended processes of care more often than expected. Second, they found that satisfaction with care was higher among clinically complex patients with high measured performance of recommended processes of care. This finding suggests that compliance with performance measures was not crowding out other aspects of care valued by these patients. This is an important finding, since as they note, if a patient has needs that lie outside of the measured conditions, performance measures may 'crowd out' unmeasured care by creating incentives for physicians to attend to measured medical problems over unmeasured ones.

Published online October 4, 2008
This line of research, however, raises a number of technical issues particularly germane to complex patients and highlights many of the clinical management issues facing such patients and their physicians. We will address a number of the technical issues and then suggest an approach to such patients that we believe will ultimately prove to be of increased value to both the patient and their physicians.

The first technical question is the mechanisms by which increased medical visits influence both quality of care and patient satisfaction. As the authors hypothesize, does seeing a single physician frequently give that doctor more opportunities to successfully address all the many competing demands of clinical complexity and still meet performance measures? It is also possible that this observed effect is mediated by seeing multiple physicians, each of whom has a different area of clinical emphasis and potential to 'catch' omissions of other doctors. Another possibility is the role of clinical systems beyond the actions of individual physicians. The Veterans Affairs (VA) health system studied in this paper is in the forefront of developing systems to monitor chronic conditions and ensure, through electronic health record alerts, mandatory intake nurse check lists, and other methods that patients with those conditions receive the necessary screening, treatment, and follow-up. Maybe this is a success of more frequent interaction with the infrastructure we are trying to institute under the Advanced Medical Home concept- and not just increased interactions with the physician. As with all good research the results often trigger many more important questions.

The next technical question is the definition of clinical complexity itself. In Werner and Chang's paper they defined clinical complexity by the anticipated cost of caring for patients with specified diagnoses using the listed patients' aggregated diagnoses in the Diagnostic Cost Groups (DCG) Hierarchical Condition Categories model ${ }^{4}$. This model uses diagnoses to develop an "expected" cost based on patients with similar diagnoses. This expected cost definition of clinical complexity might not be congruent with other potential definitions of complexity. Perhaps patients who have frequent clinic visits have more diagnoses documented and therefore have higher complexity or "expected" cost. Or perhaps documenting the diagnosis leads to screening or therapeutic "next steps". The same issue would be evident whether the definition of complexity was cost or number of diagnoses identified. The act of having more visits may indicate either more clinical need or it might just result in more documentation leading to a higher apparent clinical complexity.

The issue of circularity in definitions of clinical complexity and performance measures may seem a little persnickety, but 
the results of one of the main outcomes in the Werner article, patient satisfaction, runs counter to a body of literature exploring the relationship between other important indications of disease burden, a patient's self-reported health and functional status, and satisfaction with care. In most prior studies, including those in VA settings, patients with lower selfreported health and functional scores have been shown to have lower global satisfaction with their medical care ${ }^{5-7}$. The author's definition of clinical complexity did not incorporate patients' own assessments of their health and functional status. Perhaps these differences in results can be partially explained by the circularity of relying on diagnoses both to define the complexity of the cases and the outcomes to be assessed.

It is indeed reassuring that compliance with current performance measures did not negatively impact clinically complex patients' global satisfaction with their health care received at the VA. Yet, still unanswered is whether these performance measures indeed are capturing the most clinically relevant and highest-priority areas for these clinically complex patients. The difficulty in exploring the important domains of quality and satisfaction among patients with chronic conditions is compounded by the reliance on simplistic and clinically irrelevant and/or potentially harmful "standards of care" ${ }^{8,9}$. If we are to move to a clinically nuanced understanding of high quality care we must move beyond the every patient is a "747 awaiting takeoff" mentality. The one-size-fitsall checklist-driven approach that quality and patient safety groups have embraced may in some cases be detrimental to both quality and patient safety ${ }^{10}$. Patients with multiple conditions have multiple and interacting conditions. For example, the ACE inhibitor for a patient's heart failure (HF) might not be the optimal choice in the face of their concomitant chronic renal insufficiency, even though it is on the checklist of process measures for patients with HF. When confronted with a patient with five or six interacting chronic conditions, how do we prioritize from among the six different checklists of "quality process measures" and still have the patient (or us) understand how best to improve their health until their next visit? The real question is, "Given this patient's multiple conditions and the current evidence on these conditions, how can we optimize the patient's global health state today?" "How do we help this patient prioritize from among the many issues and focus on the subset that will be of most value to her?" This can't be found on a checklist. It can, however, be found in the voluminous clinical information available for most patients with severe and multiple chronic conditions.

To best help our patients with clinically complex conditions, we need to move from a checklist filled with "to dos" and simplistic dichotomous cut-offs that identify clinical "success" or "failure" to longitudinal, weighted measures that give more credit for high-priority care than low-priority care and are personalized for each patient. We now have the statistical tools, and emerging information infrastructure in health systems such as the VA and other integrated systems, to create multivariate risk-stratified profiles of medical conditions ${ }^{11-13}$. We can then use these models and the patient's own diagnoses, medications, visits, and laboratory values, as well as history of prior treatment intolerances and failures, to develop an action list for each patient, for each visit. We are beginning to see the results of such analyses. For example, this approach has demonstrated the importance of blood pressure control and its influence on cardiovascular risk in adults with diabetes $^{14}$.

By focusing on a select number of high-value interventions, one can better focus within the 15-minute outpatient visit on items that are both of most clinical importance and to address the preferences of clinically complex patients. This approach can at the same time that it optimizes care minimize both the cost and unintended consequences of the checklist-based, one-size-fits-all approach. The development of such measures incorporating clinically-detailed information will require political will and resources to create the necessary integrated medical information systems in many health care settings. Werner and Chang rightly highlight the crucial question of whether current performance measurement is crowding out areas of most concern to clinically complex patients. It is of equal importance to ensure that current performance measures are not 'crowding out' areas of higher clinical priority for individual patients than what is currently measured.

In summary, the paper by Werner and Chang makes an important contribution to the growing literature on quality of care and satisfaction measurement among clinically complex patients. We point out some of the current difficulties with this field both operationally and conceptually. We highlight the need for a paradigm shift in the field, to make use of the wealth of data, information systems, and statistical methods to guide the care of each of our clinically complex patients. In the era of value-based health care, we must take the lead and ensure that our sickest patients receive our most informed and directed care. We must move from a uniform list of "to dos" to a plan of directed improvement, tailored to each of our clinically complex patients. Care that targets the highest priorities for each patient needs to be the new definition of quality of care for our clinically complex patients!

Corresponding Author: Laurence F. McMahon, Jr., MD, MPH; 300 North Ingalls Ann Arbor, MI 48109, USA (e-mail: Imcmahon@umich. edu).

\section{REFERENCES}

1. Werner RM, Chang Vw. The relationship between measured performance and satisfaction with care among clinical complex patients. JGIM. doi:10.1007/s11606-008-0734-6.

2. Higashi T, Wenger NS, Adams JL, et al. Relationship between number of medical conditions and quality of care. NEJM. 2007;356(24)2496504.

3. Min LC, Wenger NS, Fung C, et al. Multimorbidity is associated with better quality of care among vulnerable elders. Med Care. 2007;45(6) 480-8.

4. Rosen AK, Loveland S, Anderson JJ. Applying diagnostic cost groups to examine the disease burden of VA facilities: Comparing the six "Evaluating VA costs" study sites with other VA sites and Medicare. Med Care. 2003;41;6(Suppl II)91-102.

5. Ren XS, Kazis L, Lee A, Rogers W, Pendergrass S. Health status and satisfaction with health care: a longitudinal study among patients served by the veterans health administration. Am J Med Qual. 2001;16(5)16673.

6. Plomondon ME, Runsfeld JS, Humble CG, et al. Factors influencing risk-adjusted patient satisfaction after coronary artery bypass grafting. Am J Cardiol. 2003;92:206-8. 
7. Kerr EA, Smith DM, Kaplan SH, Hayward RA. The association between three different measures of health status and satisfaction among patients with diabetes. Med Care Res Rev. 2003;60(2)158-77.

8. Tinetti ME, Bogardus ST, Agostini JV. Potential pitfalls of diseasespecific guidelines for patients with multiple conditions. NEJM. 2004;351(27)2870-4.

9. Boyd CM, Darer J, Boult C, Fried LP, Boult L, Wu AW. Clinical practice guidelines and quality of care for older patients with multiple comorbid diseases; implications for pay for performance. JAMA. 2005;294(6)71624.

10. Hayward RA. All-or-nothing treatment targets make bad performance measures. American Journal of Managed Care. 2007;13(3) 126-8.
11. Kent DM, Hayward RA. Limitations of applying summary results of clinical trials to individual patients: the need for risk stratification. JAMA. 2007;298(10) 1209-12.

12. Hayward RA, Kent DM, Vijan S, Hofer TP. Reporting clinical trial results to inform providers, payers and consumers. Health Aff. 2005;24 (6) $1571-81$.

13. McMahon LF Jr, Hayward RA, Saint S, Chernew ME, Fendrick AM. Univariate solutions in a multivariate world; can we afford to practice as in the "good old days"? American Journal of Managed Care. 2005; 11(8)473-6.

14. Schmittdiel J, Vijan S, Fireman B, Lafata JE, Oestreicher N, Selby JV. Predicted quality-adjusted life years as a composite measure of the clinical value of diabetes risk factor control. Med Care. 2007;45(4)315-21. 